\title{
Root Canal Treament of Maxillary Molar With Second Mesiobuccal Canal - A Case Report
}

\author{
Kurnia Fitriningtyas ${ }^{1}$, Ema Mulyawati ${ }^{2}$, Margareta Rinastiti ${ }^{2}$ \\ ${ }^{1}$ Conservative Dentistry Specialty Program, Faculty of Dentistry, Universitas Gadjah \\ Mada, Indonesia \\ ${ }^{2}$ Department of Conservative Dentistry, Faculty of Dentistry, Universitas Gadjah Mada, \\ Indonesia \\ *Coresponding author email : tyasdentist@gmail.com \\ Authorsemail:emamulyawati@ugm.ac.id,rinastiti@ugm.ac.id
}

\begin{abstract}
An insight into the complexity of internal dental morphology is an important point for the planning and performing of root canal treatment. The undetected anatomical variations existing in the root canal system may contribute to the failure of root canal therapy. An additional root canal on the mesiobuccal root is one of the morphological variations of the maxillary first molar. A 25-year-old male patient was reported to the Dental Hospital of Universitas Gadjah Mada with complaints of a big cavity in upper left first molar teeth. The tooth was not endodontically treated and there was no pain detected during the examination. Clinical examination revealed a pulp depth of distopalatoocclusal carious lesion with intrinsic discolouration. The second mesiobuccal root canal was detected during exploration. Preoperative radiographs showed pulp exposure and periradicular radiolucency. Diagnosis of tooth 26 was profound caries with necrotic pulp, asymptomatic apical periodontitis and intrinsic discolouration. Root canal treatment was carried out followed by prefabricated fibre post and porcelain fused to metal crown. Exploring the possibility of root canal variation would enhance the success of root canal treatment. The accurate instrumentation would help in the recognition and treatment of the second mesiobuccal canal.
\end{abstract}

Keywords: root canal treatment, second mesiobuccal canal, upper first molar

\section{INTRODUCTION}

An insight into the complexity of internal dental morphology is a basic requirement to achieve success during root canal procedures. It has been demonstrated by previous studies that the effect of natural variations in root canal geometry is greater than the effect of instrumentation techniques during root canal enlargement and shaping in order to achieve successful root canal treatment [1].

Failure of root canal treatment can be attributed to several factors, one of which is unprepared missed canals [1,2]. Maxillary first molars generally have three-roots with additional canal located in the mesiobuccal root [3]. The mesiobuccal root of maxillary first molars is one of the most complex root canal systems [4] and one of the most frequently studied roots both in vitro and in vivo [3,5]. The canal configuration can vary in some cases because for numerous reasons like developmental anomalies, hereditary factors, and trauma. Variation in tooth development could be an additional root canal. In fact, the most common reason for retreatment of maxillary molars is the occurrence of the missed second mesiobuccal canal [6].

\section{CASE REPORT}

A 25-year-old male patient was reported to the Dental Hospital of Universitas Gadjah Mada with complaints of a big cavity in upper left first molar teeth. The tooth was not endodontically treated and there was not painful during the examination. Clinical examination revealed a distopalatoocclusal carious lesion on tooth 26 at the depth of pulp chamber with intrinsic discolouration. The second mesiobuccal root canal was detected during exploration. 


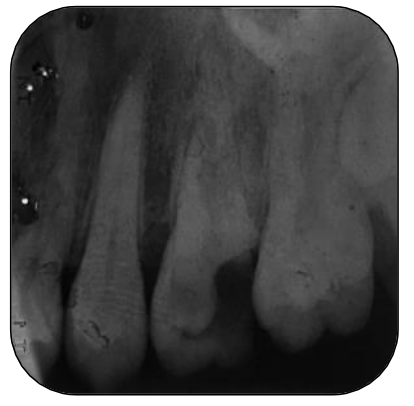

Fig. 1. Preoperative radiographs of 26

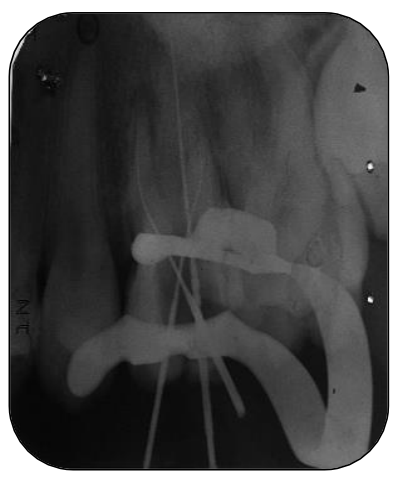

Fig. 3a. Working length determination radiograph for the mesiobuccal canal, the distobuccal canal, the palatal canal.

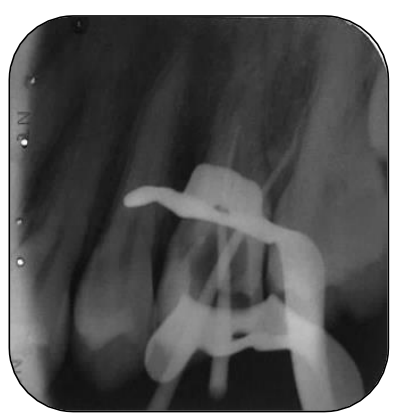

Fig. 4a. Master cone fit radiograph showing mesiobuccal canal, and distobuccal canal.

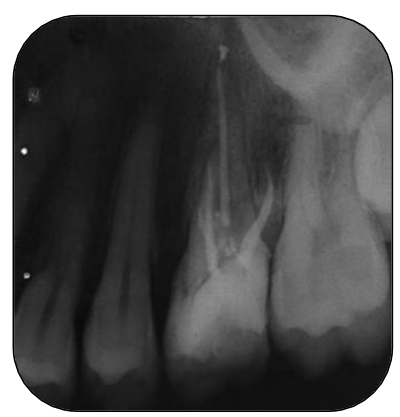

Fig. 5. Post obturation radiograph.

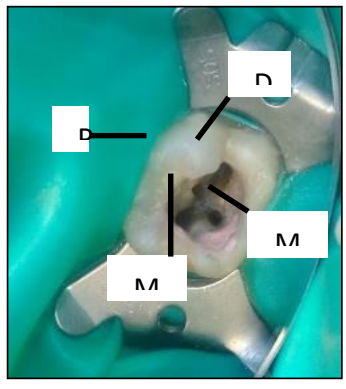

Fig. 2. Pulpal floor of 26.

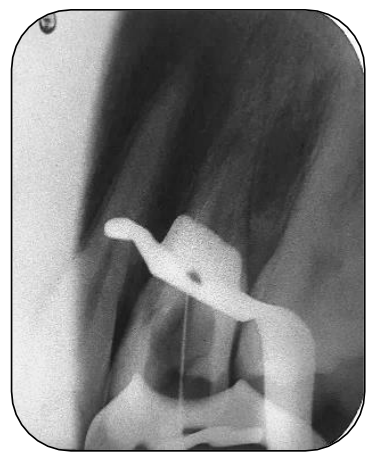

Fig. 3b. Working lengt palatal canal was prepared for F3 size. Root canals determination radiograph for $7 \%$ EDTA to remove smear layer and $2 \%$

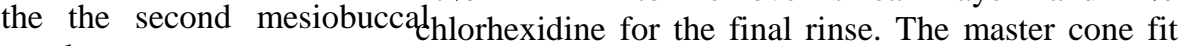
canal.

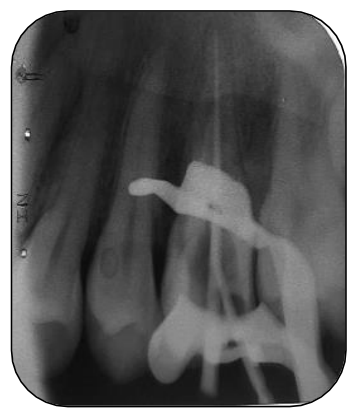
of each canal was inserted and was verified radiographically (Fig. 4a and $4 \mathrm{~b}$ ). The canals were then dried using absorbent paper points and obturated using single cone technique with guttapercha and epoxy resin sealer (Fig. 5). After completion of root canal treatment, the prefabricated fibrepost was inserted and porcelain fused to metal crown was placed. The patient has recalled for follow-up after 2,5 months and the tooth was asymptomatic (Fig. 6).

\section{DISCUSSION}

More than 70 until $97 \%$ of maxillary first molar showed the occurrence of the second mesiobuccal Fig. 4b. Master cone fitanal [2,6] and this condition is similar for all of the radiograph showing second thnic groups [1]. In vitro studies indicated that a mesiobuccal canal and palatalecond canal is present in up to $90 \%$ of maxillary first canal.

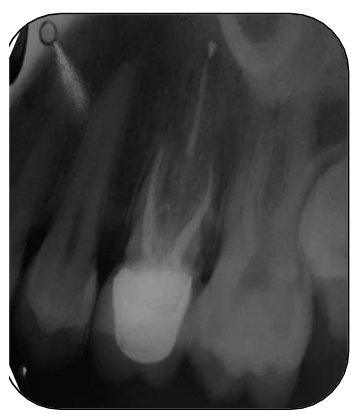

Fig. 6. Follow up at months. molars teeth [3], while in vivo studies reported a lower prevalence of the second mesiobuccal canal $(77,2 \%$ $79,8 \%$ ) [3,4]. An ex vivo micro-computed tomography study showed a similar result with up to $80 \%$ incidence of two canals in the mesiobuccal root [4].

The second mesiobuccal canal could be immediately detected in $70 \%$ of the maxillary first molar, once the pulp chamber is exposed [2]. The rest of the second mesiobuccal were observed at deeper levels in the root and this requires careful probing in the pulp floor [2]. There are several type canal 2, 5onfiguration of mesiobuccal root canals in the maxillary first molar, $40-45 \%$ of them has one canal[1,7], $37 \%$ of them has two orifices and one 
foramen apical, and $18 \%-48 \%$ of them has two canals $[1,3,7]$. The palatal and distobuccal roots usually present one canal configuration [3].

The difficulty in canal identification can be related directly to the variation and complex morphology in the mesiobuccal root of maxillary molars [2]. The second canal in mesiobuccal roots of maxillary molars is fine in size and its close proximity to larger canal makes it difficult to identify [3,8]. The second mesiobuccal canal may also join the larger canal and its orifice may be concealed by dentin lip, therefore it needs to be removed to detect the orifice [3].Coronal preenlargement as much as half to two-thirds of root canal (called coronal flaring) using Gates Glidden (recommended used for both step-back and step-down sequences) and NiTi rotary instrument (recommended used in a crown-down sequence) secures unimpeded access of file to the apical one third [1].

A thorough insight of tooth morphology, careful interpretation of radiographic documentation, and adequate access and exploration with a sharp endodontic explorer is needed to locate canal orifices and to determine their angle of departure from the pulp chamber. The operator could use several aids in determining the location of root canal orifices, including multiple pretreatment radiographs, CBCTs, an examination of the pulp chamber floor with a sharp explorer and visual assessment regarding colour changes of the dentin [1]. Another aid might be provided by throughing of anatomic grooves with ultrasonic tips, staining the chamber floor with $1 \%$ methylene blue dye, performing a sodium hypochlorite "champagne bubble" test [1,5], visualizing the pulp chamber anatomy from established documents and canal bleeding points [1].

Regarding visual identification, sequential application of $7 \%$ aqueous ethylene diamine tetraacetic acid (EDTA) and 95\% ethanol has been recommended for effective cleaning and drying of the pulp chamber floor before the visual inspection. Use an endodontic microscope may be helpful to locate second mesiobuccal canal in maxillary molars, but this only makes a small difference to the success rates associated with mesiobuccal roots when a periapical lesion was present [1]. However, there was no difference in canal counts for original and repeat reads by the two observers with digital periapical and conebeam computed tomography (CBCT), then between $\mu \mathrm{CT}$ canal counts and $\mathrm{CBCT}$, but $\mu \mathrm{CT}$ canal counts were significantly different from digital periapical radiograph [9].

The only way a clinician could effectively and safely determine the exact number of orifices in a tooth without causing iatrogenic destruction is to visualize to the full extent of pulp chamber floor by using a variety of anatomic landmarks [10]. There are three laws could be used to identify the location of orifices on pulp chamber floor including in maxillary molars. The first law is law of colour change that explain the colour of the pulp chamber floor is always darker than the walls $[1,10]$. The second law is law of orifice location 1 that explain the orifice of the root canals are always located at the junction of the walls and the floor. The third law is law of orifice location 2 that explain the orifice of the root canals are located at the vertices of the floor-wall junction [10].

The Law of Orifice Location 1 and 2, in conjunction with the Law of Colour Change, is often the only reliable indicator of the presence and location of second canals in mesiobuccal roots of maxillary molars. All of the Law of Orifice Location can be used to identify the exact position and number of orifices after the floor-wall junction is clearly seen. Considering the pulp anatomy of maxillary molars, there is an angle in the floor geometry between the mesiobuccal and palatal orifices along the floor-wall junction[10]. The second mesiobuccal canal, if present, lies mesial to a line joining the main mesiobuccal and palatal canal orifices $[3,10]$ or found just palatal to the orifice of the main mesiobuccal canal [8] or closer to mesiobuccal canal in more than $54 \%$ of the cases [5] and perpendicular from the distobuccal canal [6]. The second mesiobuccal canal could join the main mesiobuccal canal and has a single portal of exit.The chances for locating second mesiobuccal decreases as the age increases. The usual distance between main mesiobuccal and second mesiobuccal ranges from 1,5 to $2 \mathrm{~mm}$ mesially and 2,5 to $3,5 \mathrm{~mm}$ palatally with a maximum reported distance of $4 \mathrm{~mm}$. The second mesiobuccal canal was located 5,607 $\mathrm{mm}$ away from the mesiobuccal canal at the orifice level. The clinician must be aware of such an unusual location of second mesiobuccal and hence the search must be conducted even closer to the palatal orifice if the second mesiobuccal is not identified in its usual location [5].

The mesiobuccal canals are usually the most challenging to the instrument as they leave the pulp chamber in a mesial direction under a dentin shelf before curving distopalatally [1,3]. An instrument must be entered from the distal and buccal directions in order to transverse the canal. With the course of the canal being toward the buccal aspect as it approaches the apex, it is suggested that a slight buccal curve be placed in the exploring instrument [8].

In this case, the operator determined the exact number of orifices in a tooth by using a variety of anatomic landmarks to visualize to the full extent of pulp chamber floor, that is The Law of Orifice Location 1 and 2, in conjunction with the Law of Colour Change. Adequate access and exploration with a sharp endodontic explorer was needed to locate canal orifices and to determine their angle of departure from the pulp chamber.

\section{CONCLUSION}

Exploring the possibility of root canal variation would enhance the success of root canal treatment. 
The clinician should always assume that there are two canals in the mesiobuccal root until it is proven that there is only one when performing root canal treatment on maxillary first molars. The accurate instrumentation would help in recognition and treatment of second mesiobuccal canal.

\section{REFERENCES}

[1] K.M. Hargreaves, L.H. Berman, Cohen's Pathways of the Pulp, first ed., Elsevier, St. Louis, 2016, pp. 44, 130-131, 137-139, 235, 497.

[2] K. Alfouzan, A. Alfadley, L. Alkadi, A. Alhezam, A. Jamleh, Detecting the Second Mesiobuccal Canal in Maxillary Molars in a Saudi Arabian Population : A Micro-CT Study, Hindawi Scanning, (2019) 1-6.

[3] B.S. Chong, Harty's Endodontics in Clinical Practice, sixth ed., Elsevier China, 2010, pp. 43-45.

[4] R.A. Degerness, W.R. Bowles, Dimension, Anatomy and Morphology of the Mesiobuccal Root Canal System in Maxillary Molars, JOE, 36 (6) (2010) 985-989.

[5] R. Randhya, S. Sundar, D. Kowsky, N. Velmurugan, B. Arul, Unusual Location of a Second
Mesiobuccal Canal in the Maxillary First Molar managed with the Aid if Cone-beam Computed Tomography, Journal of Operative Dentistry and Endodontics, 1 (1) (2016) 35-38.

[6] N. Garg, A. Garg, Textbook of Endodontics, third ed., Jaypee Brothers Medical Publishers, New Delhi, 2014, pp. 174-175.

[7] A.A. Habib, Anatomical study of the mesiobuccal root in maxillary first molars, Journal of Taibah University Medical Sciences, 9 (2) (2014) 123125.

[8] F.S. Weine, H.J. Healey, H. Gerstein, L. Evanson, Canal Configuration in the Mesiobuccal Root of the Maxillary First Molar and Its Endodontic Significance, JOE, 38 (10) (2012) 1305-1308.

[9] J.D. Domark, J.F. Hatton, R.P. Benison, C.F. Hidebolt, An Ex Vivo Comparison of Digital Radiography and Cone-beam and Micro Computed Tomography in the Detection of the Number of Canals in the Mesiobuccal Roots of Maxillary Molars, JOE, 39 (7) (2013) 901-905.

[10] American Association of Endodontists, Endodontics Colleagues for Excellence : Access Opening and Canal Location, American Association of Endodontists, Chicago, 2010, pp. 1-8 